Lupus systemic erythematosus is characterised by an increasing risk of premature cardiovascular disease (CVD). CVD is one of the most common causes of death in SLE. Subclinical atherosclerosis in comparison to general population is also more prevalent.

Methods A cross-sectional study was carried out from March to November 2015. Patients $(119 ; 94.1 \%$ women $)$ were recruited from consultation at the Systemic Autoimmune Diseases Unit for a routine medical check.

The population was divided into three groups: patients with lupus free cardiovascular disease (free CVD), lupus with subclinic cardiovascular disease (sub CVD) (with endothelial dysfunction in ultrasonography but no clinic events) and people with clinical cardiovascular disease.

Patients with subclinical disease have higher cholesterol levels when compared to patients with established cardiovascular disease ( $p$ value $=0.008$ ) and to patients with SLE without vascular disease $(p$ value $=0.043)$. This is due to the lack of knowledge of subclinical damage by the clinician and, in addition to the absence of visible vascular damage, strict control of cholesterol levels is not performed in this group. Likewise, LDL cholesterol levels are elevated in the same context as triglycerides (differences between SLE free and SLE with subclinical SLE).

There were no differences in the activity index, what is likely to be in relation to the low activity they had at the time of inclusion in the study. We do have indeed detected differences in the time of evolution of those who present vascular damage (longer evolution time, 22.88 years) compared to patients without vascular involvement (12.32 years) suggesting that at the same time as more years of disease are accumulated vascular damage accumulates. Likewise patients with vascular damage and subclinical vascular damage were older (55.12 and 54.29, respectively), compared to those with no involvement (42.94 years).

No differences were detected between the use of antimalarials or the deleterious effect of corticosteroids and immunosuppressants (traditionally associated with increasing vascular risk) in the presence of clinical, subclinical or absence of vascular damage.

It is important to know the lupic population with a higher tendences to have vascular damage for the purpose of greater control.

\section{PS3:64 CLINICAL FEATURES OF ANTI-RNP POSITIVE PATIENTS IN KOREA}

SH Chang, SW Lee. Soonchunhyang University College of Medicine Department of Internal medicine, Chenonan Hospital, Cheonan, South Korea

10.1136/lupus-2018-abstract.110

Introduction Anti-RNP antibody is associated with overlap syndrome, which is classified either systemic lupus erythematosus (SLE) or systemic sclerosis (SSc) later. The aim of current study is to investigate whether certain clinical features of antiRNP positive patients can predict further disease predisposition.

Method We reviewed medical records of patients visited at Soonchunhyang University Cheonan Hospital since April of 1990. Initial clinical symptoms and its' onset, other clinical symptoms developed during observational periods, autoantibody profiles were investigated.
Results A total of thirty-two patients with anti-RNP positive patients were identified during 2008 to 2017 (female $n=28$, male $n=4)$. The most common initial clinical symptoms were musculoskeletal symptoms including arthritis, arthralgia, myositis, myalgia, and puffy hands $(n=10,31.1 \%)$, followed by mucocutaneous symptoms including photosensitivity, malar rash $(n=9,28.1 \%)$, cytopenia including hemolytic anaemia, leucopenia, and thrombocytopenia $(n=8,25 \%)$, Raynaud's phenomenon (RP, $n=7,21.9 \%)$, renal involvement $(n=3$, $9.4 \%)$, serositis $(n=3,9.4 \%)$, and enteritis $(n=2,6.3 \%)$. Average 9 years of follow-up duration, $87.5 \%$ of patients were diagnosed SLE $(n=28)$ while $18.8 \%$ of patients were diagnosed SSc $(n=6)$. None of patients diagnosed SSc showed initial clinical symptoms such as enteritis, serositis, cytopenia, or renal involvement. Patients who had RP at the beginning showed significantly high risk for developing SSc (Risk ratio 7.1 95\% confidential interval 1.6-31.3). Patients with positive anti-dsDNA $(n=17,53.1 \%$ of all patients, $p=0.004)$ and antiSm $(n=17, \quad 56.3 \%, \quad p=0.03) \quad$ anti-Ro/SSA $\quad(n=16, \quad 50 \%$, $\mathrm{p}=0.07$ ) antibody were significantly frequently diagnosed as SLE during follow-up periods. higher in SLE than those with SSc, while patients with SSc. Of six patients with SSc, three patients $(50 \%)$ showed anti-Scl $t$ antibody, one patient (16.7\%) showed anti-centromere antibody, and, of note, two patients $(33.3 \%)$ showed no SSc specific antibody.

Conclusion In patients with anti-RNP positive, patients with Raynaud's phenomenon have increased risk of developing systemic sclerosis even without SSc specific autoantibodies, while patients with serositis, cytopenia, enteritis, or renal involvement have higher probability to have SLE.

\section{PS3:65 VITAMIN D DEFICIENCY IN PATIENTS WITH SYSTEMIC LUPUS ERYTHEMATOSUS AND RELATIONSHIP WITH DISEASE ACTIVITY}

${ }^{1} \mathrm{~L}$ Athanassiou, ${ }^{2}$ I Kostoglou-Athanassiou, ${ }^{3} \mathrm{C}$ Katsavouni, ${ }^{3} \mathrm{~A}$ Tzanavari, ${ }^{4} \mathrm{~A}$ Koteli, ${ }^{1} \mathrm{C}$ Mavragani, 'M Koutsilieris, ${ }^{3} \mathrm{P}$ Athanassiou. ' Department of Physiology, Medical School, University of Athens, Greece; ${ }^{2}$ Department of Endocrinology, Asclepeion Hospital, Voula, Athens, Greece; ${ }^{3}$ Department of Rheumatology, St. Paul's Hospital, Thessaloniki, Greece; ${ }^{4}$ Department of Microbiology and Immunology, St. Paul's Hospital, Thessaloniki, Greece

\subsection{6/lupus-2018-abstract.111}

Purpose Systemic lupus erythematosus (SLE) is a multisystem autoimmune disorder which mainly affects women in the reproductive age. Vitamin D appears to have an immunomodulatory action, low levels of vitamin D having been described in patients with autoimmune rheumatic diseases. The aim was to report vitamin D levels and their relationship with disease activity in a cohort of SLE patients.

Methods A cohort of 20 patients, 18 female and 2 male is described. The patients were diagnosed with SLE and had arthralgias, cutaneous manifestations, fatigue and decreased complement levels. Blood levels of $25(\mathrm{OH}) \mathrm{D} 3$ were measured in all patients. $25(\mathrm{OH}) \mathrm{D} 3$ levels were measured by radioimmunoassay. The measurement of $25(\mathrm{OH}) \mathrm{D} 3$ by radioimmunoassay consisted of a two-step procedure. The first step involved a rapid extraction of $25(\mathrm{OH}) \mathrm{D}$ and other hydroxylated metabolites from serum or plasma with acetonitrile. Following extraction, the treated samples were then assayed by competitive RIA using an antibody with specificity to $25 \mathrm{OHD}$. The sample, antibody and tracer were incubated for $90 \mathrm{~min}$ at 20-25 0C. Phase separation was accomplished after $20 \mathrm{~min}$ incubation at 20-25 $0 \mathrm{C}$ with a second antibody precipitating 
complex. To reduce non-specific binding buffer was added after this incubation prior to centrifugation. The sensitivity of the assay was $<1.6 \mathrm{ng} / \mathrm{ml}$. The recovery was approximately $100 \%$ for $25(\mathrm{OH}) \mathrm{D} 3$. Within and between batch precision was $<12 \%$ and $<11 \%$, respectively.

Results In the cohort of SLE patients low blood levels of 25 (OH)D3 were observed. A positive relationship between 25 (OH)D3 blood levels and complement levels was observed, namely low 25(OH)D3 levels were positively correlated with low complement levels. An inverse relationship was observed between 25(OH)D3 levels and disease activity, namely low 25 $(\mathrm{OH}) \mathrm{D} 3$ levels were related with high disease activity.

Conclusions Vitamin D is a hormone directly related to the regulation of the musculoskeletal system. Vitamin D also has extraskeletal actions. The immunomodulatory action of vitamin $\mathrm{D}$ appears to be a key action of the hormone. In the work presented herein low blood levels of vitamin D were observed in SLE patients which were positively related to complement levels and inversely related to disease activity.

\section{Poster session 4: APS, family planning, fertility, pregnancy and neonatal care}

\section{PS4:66 PROLONGED EXPOSURE TO ANTIPHOSPHOLIPID ANTIBODIES IS ASSOCIATED WITH ENDOTHELIAL DYSFUNCTION IN PATIENTS WITH SYSTEMIC LUPUS ERYTHEMATOSUS}

${ }^{1} \mathrm{C}$ Cho, ${ }^{2} Y$ Park, ${ }^{1}$ I Baek, ${ }^{3}$ W Kim. ${ }^{1}$ Yeouido St. Mary's Hospital, Seoul, South Korea; ${ }^{2}$ St. Vincent Hospital, Suwon, South Korea; ${ }^{3}$ Seoul St. Mary's Hospital, Seoul, South Korea

\subsection{6/lupus-2018-abstract.112}

Objective Antiphospholipid syndrome has been shown to be associated with increased cardiovascular mortality, but the role of antiphospholipid antibodies (aPL) on endothelial dysfunction remains elusive. We investigated the association between endothelial dysfunction and aPL in systemic lupus erythematosus (SLE) patients.

Methods 188 SLE patients and 62 controls were enrolled. Endothelial function was measured by flow-mediated dilatation (FMD). Cardiovascular risk factors were assessed and quarterly measurement of anti-cardiolipin ( $\mathrm{aCL}$ ) and anti- $\beta 2$ glycoprotein I $\mathrm{Ab}$ were used to calculate time-integrated values throughout disease duration. Circulating endothelial progenitor cell (EPC), defined by CD34+/ KDR + mononuclear cells, was quantified by flow cytometry.

Results Median FMD was significantly lower in SLE patient than in controls $(6.9$ versus $9.3 \%, \mathrm{p}<0.001)$. In univariate analysis, older age, hypertension and persistent positive lupus anticoagulant (LAC) were associated with decreased FMD in SLE patients $(p=0.034, p=0.011, p=0.020)$. Time-integrated aCL value (TI-aCL), but not a single value, was correlated with decreased FMD $(p=0.003)$. Multivariate analysis showed that hypertension and TI-aCL were independent factors for decreased FMD $(p=0.011, p=0.011)$; addition of positive LAC increased the adjusted probability of decreased FMD $(p=0.003)$. FMD was correlated with EPC number $(\rho=0.342$, $\mathrm{p}=0.005)$ and TI-aCL was also an independent factor of reduced EPC after multiple adjustment $(p=0.024)$. The predicted probability of endothelial dysfunction at median EPC level was higher in group with high TI-aCL than in group with low TI-aCL $(p=0.004)$.
Conclusion Cumulative burden of aPL was closely associated with endothelial dysfunction in SLE patients, which was mediated in part by reduction of EPC.

\section{PS4:67 LOSING ANTIPHOSPHOLIPID ANTIBODY POSITIVITY POST THROMBOSIS IN SECONDARY ANTIPHOSPHOLIPID SYNDROME}

${ }^{1} \mathrm{M}$ Khawaja, ${ }^{2} \mathrm{~L}$ Magder, ${ }^{1} \mathrm{M}$ Petri. ${ }^{1} J o h n s$ Hopkins University School of Medicine, Dept of Rheumatology, Baltimore, USA; ${ }^{2}$ University of Maryland School of Medicine, Dept of Epidemiology and Public Health, Baltimore, USA

\subsection{6/lupus-2018-abstract.113}

Objectives Loss of positivity of antiphospholipid antibodies has been observed in clinical practice post thrombosis with secondary APS. Our study aimed to define the frequency of this loss.

Methods In this prospective study, the inclusion criteria comprised of SLE patients having at least two positive aPL markers in 3 years prior to the thrombosis. Patients with at least two post-thrombosis visits were included. Positive antiphospholipid markers comprised of RVVT $>45$, aCL IgG $\geq 20$, aCL $\operatorname{IgM} \geq 20$ and aCL $\operatorname{IgA} \geq 20$. Loss of aPL was defined as being negative for these markers for all visits after the thrombosis, excluding the first visit post thrombosis. Percentages of loss of antiphospholipid markers after thrombosis was calculated. Further analyses were done for different types of thromboses (arterial vs venous). There were 17 patients with arterial and 16 patients with venous thromboses.

Results The analysis included the numbers and percentages of patients with loss of aPL after thrombosis, as shown in the table below.

Abstract PS4:67 Table 1

\begin{tabular}{|l|l|l|}
\hline aPL Measure & Proportion (\%) with loss of aPL \\
\hline \multicolumn{3}{|c|}{ Any Thrombosis } \\
\hline RVVT $>45$ & $3 / 26$ & $12 \%$ \\
\hline $\mathrm{aCL} \mid \mathrm{gG}>=20$ & $3 / 10$ & $30 \%$ \\
\hline $\mathrm{aCL} \mid \mathrm{gM}>=20$ & $3 / 9$ & $33 \%$ \\
\hline $\mathrm{aCL} \mid \mathrm{gA}>=20$ & $0 / 2$ & $0 \%$ \\
\hline \multicolumn{3}{|c|}{ Venous Thrombosis } \\
\hline RVVT $>45$ & $2 / 16$ & $13 \%$ \\
\hline $\mathrm{aCL} \mid \mathrm{gG}>=20$ & $3 / 7$ & $43 \%$ \\
\hline $\mathrm{aCL} \mid \mathrm{gM}>=20$ & $2 / 5$ & $40 \%$ \\
\hline $\mathrm{aCL} \mid \mathrm{gA}>=20$ & $0 / 1$ & $0 \%$ \\
\hline & Arterial Thrombosis \\
\hline RVVT $>45$ & $2 / 17$ & $12 \%$ \\
\hline $\mathrm{aCL} \mid \mathrm{gG}>=20$ & $1 / 9$ & $11 \%$ \\
\hline $\mathrm{aCL} \mid \mathrm{gM}>=20$ & $1 / 10$ & $10 \%$ \\
\hline $\mathrm{aCL} \mid \mathrm{gA}>=20$ & $1 / 3$ & $33 \%$ \\
\hline
\end{tabular}

Conclusions In secondary APS due to SLE, loss of aPL positivity post thrombotic event was up to $43 \%$ for anticardiolipin IgG, IgM and IgA, but only $13 \%$ for lupus anticoagulant (measured by RVVT). Loss of lupus anticoagulant positivity appears rare. Our analyses, however, are limited due to the small numbers of prospectively followed events. 